\title{
Screen Space Animation of Fire
}

\author{
Martin Guay* \\ Université de Grenoble, INRIA
}

Fabrice Colin ${ }^{\dagger}$

Université Laurentienne
Richard Egli

Université de Sherbrooke

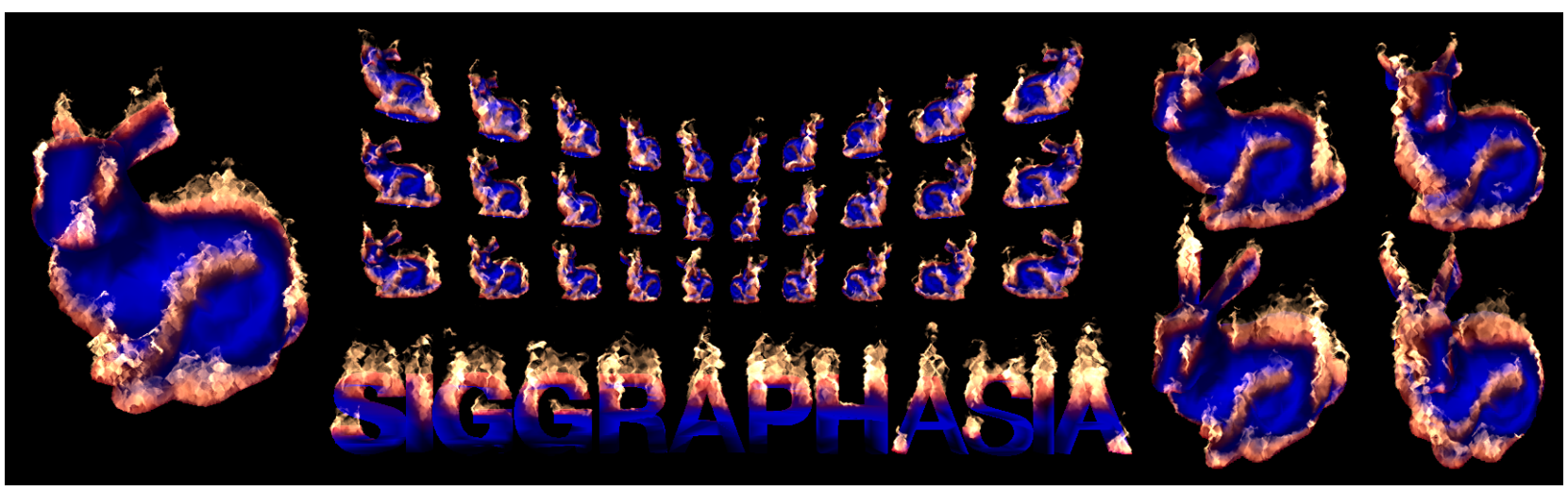

Figure 1: Ignited bunnies seen from different views. With the fire's density field ( $\phi)$ resolution set to $768 \times 768$ and the velocity field's (u) resolution set to $192 \times 192$, the animations run on the GPU at more than 1000 frames per second.

\begin{abstract}
We present a simple and physically inspired method to animate realistically looking fire directly in 2D instead of along a 3D simulation. This naturally reduces the complexity of the animation from $O\left(n^{3}\right)$ to $O\left(n^{2}\right)$. The fire is represented as a 2D scalar density field located on a plane facing the camera, and is advected under a $2.5 \mathrm{D}$ velocity field. In our method, the apparent motion of the fire on the viewing axis is mimicked by introducing vibrations in the velocity field. We model these rapid vibrations as pressure waves found in compressible fluids and therefore consider the full Navier-Stokes equations. The equations can be solved in a single pass and our method entirely runs on the GPU. A natural extension is to make use of this method directly in screen space: instead of filtering down the fire's simulation grid in world space, we rasterize the fire's source, and perform the simulation on a coarser grid directly in screen space. The results are constantly renewed 3D-looking fires computed solely in 2D.
\end{abstract}

\section{Introduction}

It was noted in [Horvath and Geiger 2009] that the motion of fire on the viewing direction is less important than the motion spanning the viewing plane. This observation led us to incorporate the motion of the fire on the third dimensional axis into a visually compelling $2.5 \mathrm{D}$ velocity field. In a similar mindset, [Rasmussen et al. 2003] obtained a three-dimensional incompressible flow by combining a set of two-dimensional simulation grids along a Kolmogorov spectrum turbulence model which provides coherence on the third dimension. Our method keeps a two-dimensional representation for the velocity, but attempts to consider the apparent motion of the fire on the third dimensional axis directly into the $2 \mathrm{D}$ velocity field. We do so by exaggerating the compressibility effects of a fluid in order to create large pressure waves, which tend to break the flames during advection and create the illusion of depth in the fire's motion.

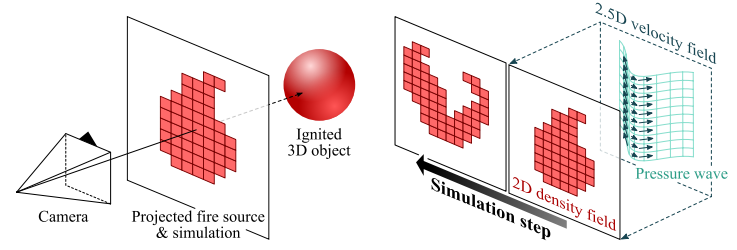

Figure 2: Left: An ignited object in the scene has its fire source value projected onto the simulation grid. Right: Pressure waves act on the shape of flames to create the illusion of $3 D$ motion.

\section{Method}

\section{$2.12 .5 \mathrm{D}$ Velocity Field}

The $2.5 \mathrm{D}$ velocity field is obtained by simulating the following compressible Navier-Stokes equations and exaggerating the compressibility effects. The mass (1) and momentum (2) conservation equations are coupled through a density-proportional pressure field (3) which we then scale in order to amplify the effects of the pressure waves:

$$
\begin{gathered}
\rho_{t}=-\nabla \cdot(\rho \mathbf{u}), \\
(\rho \mathbf{u})_{t}=-\nabla P-\rho(\mathbf{u} \cdot \nabla) \mathbf{u}+\mu \Delta \mathbf{u}+\mathbf{f},
\end{gathered}
$$

where $\rho$ is the fluid's density, $\mathbf{u}$ the velocity field, $\mu$ a diffusion coefficient, $\mathbf{f}$ external forces and $\nabla P$ the pressure gradient. The pressure field is defined as to bring the density to its rest configuration:

$$
P=k\left(\rho-\rho_{0}\right),
$$

with $\rho_{0}$ the density's initial value. As only the gradient of $P$ is of interest in equation (2), the field $P$ does not need to be stored, since $\nabla P=k \nabla \rho$. This enables us to directly link the mass (1) and momentum (2) conservation equations as follows:

$$
(\rho \mathbf{u})_{t}=-k \nabla \rho-\rho(\mathbf{u} \cdot \nabla) \mathbf{u}+\mu \Delta \mathbf{u}+\mathbf{f} .
$$

\footnotetext{
*martin.guay@inrialpes.fr

$\dagger$ fcolin@cs.laurentian.ca

† richard.egli@usherbrooke.ca
} 
The pressure field (3) was first introduced in the setting of SPH by [Desbrun and Cani 1996]. Better mass preserving alternatives have since been proposed, but which do not hold the derivative properties permitting to discard the field $P$. This feature, i.e. the coupling between both equations, allows us to formulate a single pass algorithm by solving the transport terms with the semi-Lagrangian method and the remainder with explicit central finite difference as done on the GPU for 3D incompressible fluids in [Guay et al. 2011].

The large pressure waves used to mimic the motion on the 3rd dimensional axis are created by scaling the pressure constant $k \gg$ $\frac{2 \Delta x}{\Delta t} \rho_{0}$ while keeping $\rho$ bounded: $\frac{|\Delta \rho|}{\rho_{0}}=\frac{\left|\rho-\rho_{0}\right|}{\rho_{0}}<1-\epsilon$, with $\epsilon>0$ being a small value. The large pressure fronts have the effect of breaking the flames giving the impression that they are actually modified due to the motion in the viewing direction. Keeping the $\rho$ values bounded ensures that the pressure perturbations remain local and constrained to the shape of a cellular pattern rather than becoming large global waves acting on the whole domain.

\subsection{Fire Density Advection}

The fire is represented by a 2D scalar field defined as the density $\phi$ of the fire's matter or particles. In an Eulerian formulation, this scalar field evolves with time according to the transport equation (5). This is solved with the both accurate and stable MacCormack method $\left(\approx O\left(\Delta x^{2}, \Delta t^{2}\right)\right)$ with intermediate semi-Lagrangian steps introduced in [Selle et al. 2008]:

$$
\phi_{t}=-\mathbf{u} \cdot \nabla \phi+s-c,
$$

where the constant $c$ is a global constant acting on the whole grid and $s$ is a local source term. Our velocity field holds much divergence which is not taken into account in the equation (5). This leads to greater mass loss, or greater stretching and compression in the fire's density field. We think that such effects contribute to the illusion of 3D fire as the transformations visually appear as to be caused by the fire's motion on the third dimensional axis.

\subsection{Screen Space Setting}

Instead of performing the simulation on a high-resolution 2D grid in world space and thereafter filtering down the final result with rasterization, it is possible to project only the fire's source term $s$ and perform the simulation on a coarser grid directly in screen space. Objects on fire in the scene hold a tunable value describing the intensity of the fire source. This value is rasterized along the scene and retrieved when solving (5).

We explored the possibility of using a global grid holding all the fires in screen space as opposed to individual grids for each fires. This is actually straightforward to use and allows to animate hundreds of fires simultaneously. However, as the grid stays fixed and the camera keeps moving, temporal coherency in the fire's density field must be kept throughout the simulation. In our implementation, we reproject the density field along the previous camera's transformations before proceeding with the simulation.

\section{Results}

We animate 100 fires on a global grid and compare the performance when the simulation grids are increased by a factor of 1.5 . It is important to note that the most expensive step in the simulation is the advection of the fire's density grid with the 3-pass MacCormack method due to the high resolution required for the global grid. As an example, the single pass fluid simulation alone, runs at more than 12,800 frames per second on a $256 \times 256$ grid resolution with a Geforce 9800 GT GPU. Note that the fire's density grid does not necessarily need to match the resolution of the frame buffer.

\begin{tabular}{|c|c|c|}
\hline Method & $\begin{array}{c}\operatorname{Dim}(\phi): 512 \times 512 \\
\operatorname{Dim}(\mathbf{u}): 128 \times 128\end{array}$ & $\begin{array}{c}\operatorname{Dim}(\phi): 768 \times 768 \\
\operatorname{Dim}(\mathbf{u}): 192 \times 192\end{array}$ \\
\hline \hline Simulation & $\approx 1420 \mathrm{fps}$ & $\approx 1000 \mathrm{fps}$ \\
\hline + Reprojection & $\approx 920 \mathrm{fps}$ & $\approx 670 \mathrm{fps}$ \\
\hline
\end{tabular}

\section{Discussion}

The main difference of our method with the classic stable fluids is the presence of divergence in the velocity field. The advection of a scalar field along a divergent velocity field is a key ingredient of our $2.5 \mathrm{D}$ approach. Advection along such a velocity field creates sinks and sources (holes and bumps) in the scalar field which leads to the impression of motion in the viewing direction. We believe that the animation of other gaseous phenomena such as clouds and smoke could also be accelerated with a similar 2.5D mechanism.

In this work, we explored the scalable and straightforward strategy of holding all the fires on the same global grid. This avenue led to face temporal consistency issues and in the future, we would like to investigate using only local grids for each fire. These individual simulations would no longer be computed at a constant cost, but would help alleviate much of the issues found with a global grid while still keeping the efficiency of a 2D simulation.

\section{Conclusion}

We presented a method to animate 3D-looking fire by using only a 2D grid reducing the complexity of the simulation from $O\left(n^{3}\right)$ to $O\left(n^{2}\right)$. It can be used in screen space enabling coarser grids for the simulation which increases performances furthermore. Although not physically accurate, the nonlinearities in the model provide constantly renewed fires as opposed to animations produced with precomputed textures. In the future, we would like to extend our 2.5D approach to other types of gaseous phenomena such as smoke and clouds.

\section{References}

Desbrun, M., And CANI, M.-P. 1996. Smoothed particles: A new paradigm for animating highly deformable bodies. In Computer Animation and Simulation '96, 61-76.

Guay, M., Colin, F., And Egli, R. 2011. Simple and Fast Fluids. GPU Pro 2, A. K. Peters Ltd, 433-444.

Horvath, C., And Geiger, W. 2009. Directable, HighResolution Simulation of Fire on the GPU. In Proceedings of ACM SIGGRAPH, vol. 28 .

Rasmussen, N., Nguyen, D. Q., Geiger, W., And Fedkiw, R. 2003. Smoke Simulation For Large Scale Phenomena. In Proceedings of ACM SIGGRAPH, vol. 22, 703-707.

Selle, A., Fedkiw, R., Byungmoon, K., Yingjie, L., And JAREK, R. 2008. An Unconditionally Stable MacCormack Method. Journal of Scientific Computing 35, 2-3, 350-371. 